\title{
ESTUDO COMPARATIVO ENTRE ANEURISMAS ROTOS TRATADOS POR CIRURGIA E POR VIA ENDOVASCULAR
}

\author{
Marcelo D. Conrad, Isabelle Pelissou-Guyotat, Christophe Morel, \\ Gabor Madarassy, Claudio Schonauer, Robert Deruty
}

\begin{abstract}
RESUMO - Realizamos análise comparativa dos resultados clínicos e radiológicos após o tratamento de aneurisma roto, localizado no setor anterior, por cirurgia ou por via endovascular. Entre 1995 e 1999, 78 pacientes foram tratados em nosso serviço por apresentarem ruptura de aneurisma intracraniano com grau clínico (Hunt \& Hess) variando entre I e III. Dentre estes pacientes, 52 foram operados, 21 embolizados e 5 fizeram tratamento combinado. Dos casos cirúrgicos, o resultado clínico foi considerado ótimo em $80,8 \%$ com mortalidade de $5 \%$ e oclusão completa do aneurisma em $96,2 \%$. Dos pacientes embolizados, $95 \%$ dos casos o resultado foi considerado ótimo com oclusão completa do aneurisma em 42,8\%. 0 tratamento endovascular de aneurismas intracranianos apresenta uma vantagem no resultado clínico em relação aos aneurismas operados. Porém, apresentam taxa de exclusão completa baixa e podem necessitar de tratamento complementar.
\end{abstract}

PALAVRAS-CHAVES: aneurismas intracranianos, repermeabilização, cirurgia após embolização.

\section{Comparative study for ruptured aneurysms treated by surgery and endovascular method}

\begin{abstract}
The management of intracranial aneurysms has truly evolved after the introduction of the endovascular treatment. In this paper we compare patients that were operated or embolized for intracranial aneurysms. Between 1995 and 1999, 78 grade I to III ruptured aneurysms were treated in our service: 52 patients were operated, 21 were embolized and 5 were submitted to combinated endovascular and surgical treatment. In the surgical group, clinical outcome was very good in $80.8 \%$ of cases with $5 \%$ of mortality with $96.2 \%$ of total exclusion of the aneurysm. In the endovascular group, $95 \%$ of cases the clinical outcome was very good with only $42.8 \%$ of total exclusion of the aneurysm. By the endovascular method for treatment of aneurysms, we can obtain a good clinical outcome but a poor radiological outcome and sometimes need a complementary surgical procedure to treat residual aneurysm.
\end{abstract}

KEY WORDS: intracranial aneurysms, aneurysm regrowth, surgery post-embolization.

O manejo dos aneurismas intracranianos e suas complicações tem evoluido após a introdução do tratamento endovascular por Guglielmi Detachable Coils (GDC) na década de 90. Este método é uma opção alternativa para os casos de difícil acesso cirúrgico. Os resultados do tratamento cirúrgico dos aneurismas tem melhorado após a aplicação de técnicas microscópicas e do tratamento clínico e cirúrgico precoces após a sua ruptura ${ }^{1,2}$. As técnicas do tratamento endovascular têm evoluido muito nestes últimos anos, após os primeiros relatos de Serbinenko em 1974. Isto conduziu a ampla utilização da técnica no tratamento dos aneurismas intracranianos, até mesmo em casos em que uma clipagem cirúrgica seria possível, devido ao caráter menos invasivo da embolização por GDC.
Este aumento nas indicações de tratamento por via endovascular, muitas vezes devido a um desrespeito na seleção dos critérios morfológicos do aneurisma, provoca falha técnica, necessitando de tratamento cirúrgico complementar para abordar complicação ou uma recanalização do aneurisma ${ }^{4-12}$.

Em casos de aneurismas complexos ou em pacientes em estado clínico grave, pode-se utilizar uma combinação entre as duas técnicas, principalmente na prevenção de recidiva hemorrágica pela compactação de coils no fundo do saco do aneurisma até melhora clínica do paciente para um tratamento cirúrgico ulterior ${ }^{4,5,13}$. Para obter os melhores resultados no tratamento dos aneurismas intracranianos, é fundamental a participação de uma equipe multidisciplinar na decisão terapêutica.

Hôpital Neurologique et Neurochirurgicale Pierre Wertheimer, Lyon - France.

Recebido 16 Julho 2001, recebido na forma final 28 Setembro 2001. Aceito 12 Outubro 2001.

Dr. Marcelo D. Conrad - Service de Neurochirurgie Professeur Deruty - Hôpital Neurologique - 59, Boulevard Pinel - 69003 Lyon - France. E-mail: mconrad@rocketmail.com 
Neste artigo, comparamos os resultados clínicos e radiológicos em pacientes que sofreram ruptura de um aneurisma intracraniano e apresentaram graus clínicos I a III (escala de Hunt \& Hess) e que foram tratados por cirurgia ou por via endovascular.

\section{MÉTODO}

Entre 1995 e 1999, 78 pacientes com um ou múltiplos aneurismas cerebrais rotos, em graus clínicos I a III de Hunt \& Hess, foram tratados em nosso serviço, correspondendo a um total de 82 aneurismas tratados. Os critérios analisados foram: idade do paciente, grau clínico no momento da hospitalização, morfologia e localização do aneurisma. Em todos os casos, a decisão do método terapêutico a ser utilizado era feita por equipe multidisciplinar composta por um neurocirurgião e um neurorradiologista. A decisão baseava-se na comparação entre risco / benefício de cada método.

Em todos os casos, foi realizado controle angiográfico 3 meses após o tratamento cirúrgico ou endovascular. Nos casos em que o tratamento por GDC foi realizado, o controle angiográfico foi realizado imediatamente após a embolização e 3, 6, 12 e 24 meses depois.

Os resultados dos controles angiográficos foram analisados pela mesma equipe multidisciplinar. Os critérios para análise destes resultados foram: 1. oclusão total: $100 \%$ de oclusão do aneurisma; 2 . oclusão sub-total: > 95\% de oclusão do aneurisma; 3 . oclusão incompleta: < 95\% do aneurisma.

Nos controles angiográficos dos aneurismas tratados por GDC realizados a 6, 12 ou 24 meses, as oclusões subtotais foram classificadas como estáveis (sem evolução do resíduo nos controles sucessivos) ou instáveis (recanalização ou evolução do resíduo).

Estes pacientes foram divididos em 3 grupos: Grupo I - 52 pacientes tratados por clipagem cirúrgica ou de aneurismas revestidos com teflon e cola biológica; Grupo II - 21 pacientes cujo aneurisma roto foi tratado por via endovascular com GDC; Grupo III - 5 pacientes tratados por embolização com GDC. No controle angiográfico observou-se recanalização ou instabilidade do resíduo do aneurisma, necessitando de tratamento cirúrgico complementar (Tabela 1).

Os resultados clínicos foram classificados em: 1) ótimos ou bons, sem déficit ou déficit neurológico mínimo; 2) médios, déficit neurológico moderado; 3) ruins, déficit neurológico grave; 4) óbito.

\section{RESULTADOS}

\section{Grupo I}

Foram tratados 52 pacientes: 22 homens $(42,3 \%)$ e 30 mulheres $(57,7 \%)$. A média de idade foi 47,8 anos (21 a 76 anos). Em 73\%, os pacientes apresentaram hemorragia subaracnoídea apresentando grau clínico I e II de Hunt \& Hess. Em $27 \%$, os pacientes apresentaram grau clínico III. A localização dos aneurismas foi em $42,3 \%$ dos casos na artéria cerebral média ( $A C M)$ e, em 40,3\% na artéria comunicante anterior (AcoA). Em 76,9\% dos casos o tamanho do aneurisma era inferior a $10 \mathrm{~mm}$ e, em $23 \%$ entre 10 e $25 \mathrm{~mm}$. Em 2 casos os aneurismas eram gigantes. A clipagem cirúrgica foi possível em $96,15 \%$ dos casos. Em 2 pacientes, devido à morfologia do aneurisma, utilizamos a técnica de revestimento do aneurisma com teflon e cola biológica ${ }^{14}$.

Os resultados clínicos foram: ótimos em 80,8\% dos casos, médio em 7,6\%, ruim em 5,7\%. Ocorreram 3 óbitos: 2 pacientes apresentaram uma agravação clínica devido a recidiva hemorrágica 24 horas após o primeiro episódio e, em 1 caso, por ressangramento após clipagem parcial do aneurisma.

Os resultados angiográficos confirmaram a exclusão completa do aneurisma em $100 \%$ dos casos clipados. Nos dois casos de revestimento com teflon, o controle angiográfico confirmou a mesma imagem observada antes do tratamento.

Tabela 1. Grupo III. Tratamento cirúrgico complementar em pacientes tratados previamente por embolização com GDC.

\begin{tabular}{|c|c|c|c|c|c|c|c|c|}
\hline Caso & $\begin{array}{l}\text { Idade / } \\
\text { sexo }\end{array}$ & $\begin{array}{l}\text { Apresentação } \\
\text { Inicial } \\
\text { (Hunt \& Hess) }\end{array}$ & $\begin{array}{l}\text { Localização } \\
\text { do aneurisma }\end{array}$ & $\begin{array}{l}\text { Tamanho } \\
(\mathrm{mm})\end{array}$ & $\begin{array}{c}\text { Resultado } \\
\text { pós-embolização }\end{array}$ & $\begin{array}{l}\text { Técnica } \\
\text { cirúrgica }\end{array}$ & $\begin{array}{l}\text { Resultado } \\
\text { clínico } \\
\text { (GOS) }\end{array}$ & $\begin{array}{l}\text { Resultado } \\
\text { radiológico }\end{array}$ \\
\hline 1 & $30 / F$ & II & $\mathrm{ACoP}$ & $<10$ & Sub-total & Clipagem & 5 & Oclusão completa \\
\hline 2 & $65 / F$ & II & $\mathrm{ACl}$ & $<10$ & Sub-total & Clipagem & 1 & Não realizado \\
\hline 3 & $48 / \mathrm{M}$ & 1 & ACoA & $<10$ & Sub-total & $\begin{array}{l}\text { Clipagem e } \\
\text { coagulação }\end{array}$ & 5 & Oculsão completa \\
\hline 4 & $58 / F$ & III & ACM & $<10$ & Sub-total & Coagulação & 5 & Oculsão completa \\
\hline 5 & $47 / F$ & II & ACoP & $10-15$ & Incompleta & Clipagem & 5 & Oclusão completa \\
\hline
\end{tabular}

F, feminino; $\mathrm{M}$, masculino; Idade em anos; $\mathrm{ACoP}$, artéria comunicante posterior; $\mathrm{ACl}$, artéria carótida interna; $\mathrm{ACoA}$, artéria comunicante anterior; $\mathrm{ACM}$, artéria cerebral média; GOS, Glasgow Outcome Scale; graduação do GOS: 1, óbito; 5, assintomático. 


\section{Grupo II}

Foram tratados 21 pacientes: 8 homens $(38,1 \%)$ e 13 mulheres (61,9\%\%). A média de idade foi 47,9 anos (21 a 75 anos). Em 71,4\%, os pacientes apresentaram hemorragia subaracnoídea com grau clínico I e II de Hunt \& Hess e grau clínico III em $28,6 \%$. A localização principal dos aneurismas foi em 33,3\% dos casos na ACoA e em 33,3\% dos casos na artéria carótida interna - artéria comunicante posterior ( $\mathrm{ACl}$ ACoP). Em apenas $14,2 \%$ dos casos, os aneurismas localizavam-se na ACM. Em 76,2\% dos casos o tamanho do aneurisma era inferior a $10 \mathrm{~mm}$ e em $23,8 \%$ entre 10 e $25 \mathrm{~mm}$.

Os resultados clínicos foram ótimos em $95 \%$ dos casos e médios em $5 \%$.

Os resultados angiográficos confirmaram a exclusão completa do aneurisma em apenas $42,8 \%$ dos casos. Em 33,3\% dos casos a oclusão foi sub-total e foi incompleta em 23,9\%. Nos controles angiográficos ulteriores, observou-se uma instabilidade do resíduo em 2 casos necessitando de nova sessão de embolização.

\section{Grupo III}

Foram tratados 5 pacientes: 4 mulheres $(80 \%)$ e 1 homem (20\%). A média de idade foi 49,6 anos (30 a 65 anos). $80 \%$ dos pacientes apresentaram hemorragia subaracnoídea com grau clínico I e II de Hunt \& Hess. Não houve uma localização preferencial (1 ACl, 1 ACoA, 1 ACM e 2 ACoP). Em 80\% destes aneurismas, o tamanho era inferior a $10 \mathrm{~mm}$ antes do primeiro tratamento.

Os resultados clínicos, após o tratamento endovascular, foram ótimos em $100 \%$ dos casos. Os resultados clínicos, após o tratamento cirúrgico, foram ótimos em $80 \%$ dos casos. Uma paciente faleceu 2 meses após a cirurgia. Neste caso, a complicação decorreu de placa de ateroma na origem do vaso portador, ocasionando estenose após a clipagem. No pósoperatório, a paciente permaneceu em coma seguido de óbito.

Os resultados angiográficos confirmaram a exclusão completa do aneurisma em $100 \%$ dos aneurismas clipados.

Em todos os casos operados, o saco do aneurisma era translúcido e distendido pelos coils. Em apenas 1 (Caso 3), houve ruptura do lóbulo residual do aneurisma, necessitando de uma clipagem transitória.

A clipagem do colo residual foi possível em 4 casos. Em 3 deles o colo do aneurisma estava livre do material e, no Caso $n^{\circ} 2$, a clipagem foi dificultada pela proximidade dos coils ao colo do aneurisma e da presença de uma placa de ateroma no vaso portador, causando estenose do vaso após a clipagem.

No Caso 4, a clipagem foi impossível devido a ocupação parcial do colo do aneurisma pelos coils. A cada tentativa de clipagem uma estenose importante do vaso portador era gerada. O tratamento consistiu na coagulação do resíduo do aneurisma associada a revestimento com teflon e cola biológica.

Em nenhum dos casos foi realizada a remoção do material de embolização devido aos riscos inerentes a esta técnica e a aderência do material na parede do vaso. O seguimento médio dos pacientes foi de 8 meses ( 2 meses a 2 anos).

\section{DISCUSSÃO}

As maiores mudanças no manejo dos aneurismas intracranianos têm ocorrido no setor da neurorradiologia intervencionista. A introdução desta técnica ${ }^{3}$, para o tratamento endovascular de aneurismas intracranianos propiciou uma grande evolução, principalmente após a introdução de GDC, na década de 90. O tratamento cirúrgico tem conduzido a grande melhora dos resultados clínicos após a utilização de técnicas microcirúrgicas para a clipagem dos aneurismas $^{1,2}$.

Para obter melhores resultados, a seleção criteriosa dos pacientes é extremamente importante e deve ser tomada por equipe multidisciplinar formada por um neurocirurgião com boa experiência vascular e um neurorradiologista intervencionista ${ }^{6}$. Vários são os critérios de seleção para o tratamento endovascular - idade avançada, gravidade do quadro clínico, presença de edema cerebral ou de vasoespasmo, relação colo/saco 1:39,11, localização do aneurisma, contra-indicação cirúrgica, opção do paciente, ausência de hematoma compressivo. Falhas no tratamento endovascular podem estar ligadas à utilização indiscriminada da técnica.

O tratamento incompleto dos aneurismas intracranianos pós-embolização é frequente. Lot et al. ${ }^{9}$ relatam $29 \%$ de tratamentos incompletos em sua série de 293 aneurismas embolizados. Raftopoulos et al. ${ }^{11}$ relatam $31,2 \%$ de oclusão sub-total ou incompleta em sua série de 64 aneurismas embolizados. Na série de 169 casos publicada por Cognard et al. ${ }^{15}$, em $44 \%$ deles foi verificada oclusão sub-total ou incompleta, 18 deles necessitando de tratamento endovascular complementar. A análise destas séries é de grande importância na comparação dos resultados obtidos após clipagem cirúrgica ${ }^{16,17}$. 
A recanalização dos aneurismas, após o tratamento por GDC e que necessitam de tratamento complementar, não é rara, com porcentagens variáveis ${ }^{4-}$ 13,15,18-22. Em nossa série de aneurismas rotos, tratados por GDC, encontramos 33,3\% de tratamento incompleto. Em 9,5\% dos casos, uma nova embolização foi realizada. Em 23,8\% não foi possível realizar novo tratamento endovascular, necessitando tratamento cirúrgico complementar. Na série publicada por Gurian et al. ${ }^{6}, 7,1 \%$ dos aneurismas embolizados necessitaram de tratamento cirúrgico complementar. Outros relatos de tratamento cirúrgico complementar à embolização foram descritos ${ }^{4,5}$.

O controle angiográfico rotineiro para os aneurismas tratados por GDC, principalmente nos casos onde a oclusão total do aneurisma, nem sempre foi possivel. Byrne et al. ${ }^{4}$ relatam o aumento do volume de resíduo de aneurisma com um risco hemorrágico anual de $0,8 \%$. Para os resíduos após clipagem este risco é de $0,38 \% \%^{4,23}$. Quando há tratamento incompleto de aneurismas pela técnica endovascular, e o resíduo é considerado instável, a decisão deve ser novamente tomada por uma equipe multidisciplinar. Assim como Gurian et al. ${ }^{6}$, nos casos de resíduos instáveis, preconizamos a realização de nova sessão de embolização. Nos casos em que nova embolização não é possível, uma intervenção cirúrgica deve ser realizada.

A clipagem cirúrgica dos resíduos de aneurismas nem sempre é simples de ser realizada, devido a presença de protrusão dos coils no colo, gerando risco importante de estenose do vaso portador no momento da clipagem (Casos 2 e 4). Aumenta-se o risco de morbidade e mortalidade, principalmente quando existem outros fatores que podem favorecer esta situação, como no Caso 2 de nossa série.

Alguns autores ${ }^{5,7}$ preconizam a retirada dos coils quando a clipagem do resíduo do aneurisma é dificil. Outros ${ }^{6,12}$, preconizam acompanhamento do resíduo até o momento em que o colo do aneurisma fique livre do material, facilitando a clipagem. Nossa opinião é que a retirada dos coils nem sempre é simples, devido a presença do trombo intra-aneurismático e a forte aderência do material à parede do vaso, principalmente se a embolização foi realizada após algumas semanas, podendo levar a ruptura vascular catastrófica. Em nenhum dos casos de nossa série foi retirado o material. O acompanhamento da evolução do resíduo até que o colo do aneurisma fique livre deve ser bem ponderada, pois o risco de recidiva hemorrágica não deve ser negligenciada. A coagulação do resíduo do aneurisma pode ser uma boa técnica para os casos nos quais a clipagem deste resíduo é impossível, devido a risco de estenose do vaso portador. Concordamos com a opinião com Horowitz et al. ${ }^{7}$, pois a exposição cirúrgica proximal e distal do vaso portador do resíduo do aneurisma é de importância capital para um maior sucesso no tratamento destes casos, principalmente quando a clipagem transitoria é necessária (Caso 3).

\section{CONCLUSÕES}

Para obtermos melhores resultados no tratamento dos aneurismas rotos, a decisão terapêutica deve ser tomada por uma equipe multidisciplinar composta por um neurocirurgião e um neurorradiologista.

Uma comparação entre as duas técnicas permite concluir que o tratamento endovascular dos aneurismas intracranianos é excelente método se analisarmos apenas os critérios clínicos. Em relação aos resultados radiológicos, há necessidade de uma evolução desta técnica, principalmente nos casos em que a embolização completa do aneurisma não é possível devido aos aspectos morfológicos. Em nossa série de 5 casos de resíduos de aneurismas tratados por via endovascular, necessitando de tratamento complementar, vem a cofirmar relatos da literatura.

Nos casos de recanalização de aneurismas após tratamento por GDC, encontra-se algumas dificuldades particulares na decisão terapêutica a ser tomada: observação, nova série de embolização ou tratamento cirúrgico complementar. O conhecimento da história natural e evolução destes casos é de grande importância.

A presença de resíduos de aneurismas nem sempre é simples de ser tratada cirurgicamente. A clipagem desses resíduos pode gerar estenoses do vaso portador. Nestas situações, uma coagulação cuidadosa deste resíduo pode ser técnica opcional com excelentes resultados clínicos.

Quando bem indicados, o tratamento por via endovascular ou o tratamento cirúrgico, isolados ou em associação, podem melhorar ainda mais as taxas de morbidade e mortalidade no tratamento dos aneurismas intracranianos.

\section{BIBLIOGRAFIA}

1. Deruty R, Mottolese C, Pelissou-Guyotat I, Soustiel JF. Management of the ruptured intracranial aneurysm: early surgery, late surgery, or modulated surgery? Personal experience based upon 468 patients admitted in two periods (1972-1984 and 1985-1989). Acta Neurochir (Wien) 1991;113:1-10.

2. Deruty R, Pelissou-Guyotat I, Mottolese C, Amat D. Long term outcome after treatment of the ruptured intracranial aneurysm: 73 cases admitted from day 0 to day 3 after subarachnoid haemorrhage. Neurol Res 1994;16:83-88.

3. Serbinenko FA. Balloon catheterization and occlusion of major cerebral vessels. J Neurosurg 1974;41:125-145. 
4. Byrne JV, Sohn MJ, Molyneux AJ. Five-year experience in using embolization for ruptured intracranial aneurysm: outcomes and incidence of late rebleeding. J Neurosurg 1999;90:656-663.

5. Civit T, Auque J, Marchal JC, Bracard S, Picard L, Hepner H. Neurosurgery 1996;38:955-961.

6. Gurian JH, Martin NA, King WA, Duckwiler GR, Guglielmi G, Viñuela F. Neurosurgical management of cerebral aneurysms following unsuccessful or incomplete endovascular embolization. J Neurosurg 1995;83:843-853.

7. Horowitz M, Purdy P, Kopitnik T, Dutton K, Samson D. Aneurysm retreatment after Guglielmi detachable coil and nondetachable coil embolization: report of nine cases and review of the literature. Neurosurgery 1999;44:712-720.

8. Ladouceur DL. Transcranial clipping of recurrent cerebral aneurysm after endovascular treatment. Stroke 1993;24:1087-1089.

9. Lot G, Houdat E, Cophignon J, Casasco A, George B. Combined management of intracranial aneurysms by surgical and endovascular treatment. Modalities and results from a series of 395 cases. Acta Neurochir (Wien) 1999;141:557-562.

10. Mizoi K, Yoshimoto T, Takahashi A, Nagamine Y. A pitfall in the surgery of a recurrent aneurysm after coil embolization and its histological observation: technical case report. Neurosurgery 1996;39:165-169.

11. Raftopoulos C, Mathurin P, Boscherini D, et al. Prospective analysis of aneurysm treatment in a series of 103 consecutive patients when endovascular embolization is considered the first option. J Neurosurg 2000;93:175-182

12. Thornton J, Dovey Z, Alazzaz A, et al. Surgery following endovascular coiling of intracranial aneurysms. Surg Neurol 2000;54:352-360.

13. Hacein-Bey L, Connoly Jr S, Mayer SA, Young WL, Pile-Spellman J, Solomon RA. Complex intracranial aneurysms: combined operative and endovascular approaches. Neurosurgery 1998;43:1304-1313.
14. Pelissou-Guyotat I, Deruty R, Mottolese C, Amat D. The use of Teflon as wrapping material in aneurysm surgery. Neurol Res 1994;16:224227.

15. Cognard C, Weill A, Spelle L, et al. Long-term angiographic follow-up of 169 intracranial berry aneurysms occluded with detachable coils. Radiology 1999;212:348-356.

16. Sindou M, Acevedo JC, Turjamn F. Aneurysmal remnants after microsurgical clipping: classification and results from a prospective angiographic study (in a consecutive series of 305 operated intracranial aneurysms). Acta Neurochir (Wien) 1998;140:1153-1159.

17. Thornton J, Bashir Q, Aletich VA, Debrun GM, Ausman JI, Chabel FT. What percentage of surgically clipped intracranial aneurysms have residual necks?. Neurosurgery 2000;46:1294-1300.

18. Forsting $\mathrm{M}$, Albert FK, Jansen $\mathrm{O}$, et al. Coil placement after clipping: endovascular treatment of incomplete clipped cerebral aneurysms. J Neurosurg 1996;85:966-969.

19. Fraser KW, Halbach VV, Teitelbaum GP, et al. Endovascular platinum coil embolization of incompletely surgically clipped cerebral aneurysms. Surg Neurol 1994;41:4-8.

20. Mizoi K, Takahashi A, Yoshimoto T, Fujiwara S, Koshu K. Combined endovascular and neurosurgical approach for paraclinoid internal carotid artery aneurysms. Nerosurgery 1993;33:986-992.

21. Pierot L, Boulin A, Visot A, Dupuy M, Gailard S, Derome PJ. Postoperative aneurysm remnants: endovascular treatment as an alternative to further surgery. Neuroradiology 1999;41:315-319.

22. Thielen KR, Nichols DA, Fulgham JR, Piegras DG. Endovascular treatment of cerebral aneurysms following incomplete clipping. J Neurosurg 1997;87:184-189.

23. Feuerberg I, Lindquist C, Lindqvist M, Steiner L. Natural history of postoperative aneurysm rests. J Neurosurg 1987;66:30-34. 